\title{
Design Formulas for Broadband Concentric Circular-Loops Antennas
}

\author{
Sameir M. Ali Hamed ${ }^{1 *}$ and Ashraf A. Osman ${ }^{2}$ \\ ${ }^{1}$ Nile Valley University, Sudan \\ ${ }^{2}$ Folsom CA, USA \\ *corresponding author: alihamed66@hotmail.com
}

\begin{abstract}
This paper presents a simple formulas for designing broadband concentric circular-loops antennas (CCLAs). The loop dimensions for widest bandwidth are derived and expressed in terms of driven loop resonance frequency. The analysis addresses CCLAs with and without ground planes senarios.The derived formulas are used to design multiple CCLAs. For example, a 3-elements CCLA of a maximum radius of $6.34 \mathrm{~cm}$ can be designed to operate with a voltage standing wave ratio (VSWR) $<2$ over the frequency band with fractional bandwidth of $16.9 \%$ with a minimum directivity of $3.9 \mathrm{~dB}$. Addionationally, a 4-elements CCLA of maximum radius of $15 \mathrm{~cm}$ can be design to operate in the frequency band extending from $825 \mathrm{MHz}$ to $991 \mathrm{MHz}$ (fractional bandwidth of $18.3 \%$ ) with a VSWR $<2$ and directivity higher than $5.2 \mathrm{~dB}$ over the entire band. Furthermore, the analysis shows that a 3-elements CCLA backed with a conducting ground plane improves the fractional bandwidth to $19.6 \%$ and directivity to $9.15 \mathrm{~dB}$ over the band of interest. The analytical results on designed antennas are validated with simulation results obtained by using the AN-SOF antenna simulation software. An excellent agreement is observed between analytical and AN-SOF simulations results.
\end{abstract}

Index Terms: Arrays, broadband antennas, ground plane, loop antennas.

\section{Introduction}

The circular loop antenna has attracted extensive research due to its straightforward analysis by expansion of the current as a Fourier series, as well as its simple geometry [15]. It is known that the maximum directivity of a single isolated circular loop antenna is about $4.5 \mathrm{~dB}$ and has a narrow impedance bandwidth with a reflection coefficient greater than $-6 \mathrm{~dB}$ when the loop is fed by a $50 \Omega$ line.

The directivity and/or voltage standing wave ratio characteristics of a loop antenna can be improved by using a multi-turn loop system or backing the loop with a reflectortype conducting bodies. Yagi-Uda array of circular loops can be designed for higher directivity than that of a single loop depending on array elements number and elements spacing [1]. Such an array of two elements can be optimized to give a directivity of $8 \mathrm{~dB}$ [2]. Research has shown that when circular loop antenna is backed with planar reflectors, the directivity can reach $10 \mathrm{~dB}[6,7]$. B. S. Hoon et. al. [8] have shown that when inserting concentric ring(s) within loop element, then the loop antenna impedance bandwidth can be improved. R. L. Li et. al. [9] have analyzed a discrete square multi-loop antenna system of $N$ elements where it was shown that a VSWR $<2$ bandwidth of $16 \%$ is obtained when $N=7$. On the other hand, H. Nakano et. al. [10] demonstrated that the bandwidth of circular polarization can be significantly increased when a parasitic loop is inserted inside a loop antenna. S. Hamed et. al. [11, 12] have shown that the presence of a conducting body of revolution type reflectors near a loop antenna can improve both antenna directivity and VSWR. Even though extensive research is conducted on loop antenna arrays and systems as Yagi-Uda and log periodic arrays, seldom published work on concentric loop array configuration can be found. On 1958, Schell et. al. [13] reported on a concentric array of thin-wire loops with their circumferences being multiple integers of the wavelength. Each loop is energized properly through feed pairs applied on each loop where the antenna was used for radiation pattern synthesis purpose.

The radio frequency identification (RFID) systems in the UHF bands (840 MHz - $960 \mathrm{MHz})$ have found a considerable attention recently by many researchers for their various applications [14-20]. The RFID system consists of a tag, reader antenna, and a processing unit. The desired characteristic of the reader antenna which plays a significant role in the RFID system performanceare [21]: compact size, directional with higher gain, circular polarization, good impedance match, ease to integrate, and low cost. Furthermore, it is required that these characteristics to be stable within a wide range of frequencies. The loop antenna configuration demonstrates all of the aforementioned characteristics and hence it can be projected as good candidate of an RFID reader antenna. Many researchers have reported on using loop antennas in RFID system design as reader antenna for near-field and far-field applications [1518]. The above literature indicate that a proper loop antenna design with multi-loops system with or without nearby conducting bodies can greatly enhance antenna bandwidth and directivity.

The objective of this paper is to present simple fomulas that can be used to design a broadband CCLA. Two scenarios are considered in the discussion: 1) A CCLA without a ground plane and 2) A CCLA backed with a ground plane. General design parameters for both cases are expressed in terms of an arbitrary resonance frequency. The antenna characteristics are analyzed theoretically using the wellknown circular loop antennas theory and coaxial array of circular loop antennas analysis presented in [2]. The proposed methodology is employed to design various multielement antenna and derive their characteristics. Analytical results show attractive designs that are suitable for broadband frequency range and adequate for universal UHF RFID applications. To further validate the proposed technique, the AN-SOF antenna simulator is employed to 
produce antenna design characteristics numerically. An execellent agreement between analytical and numerical simulations is observed.

The antenna geometry and theoretical development are discussed in section 2. The design technique is presented in section 3, and numerical results are discussed in section 4 . A brief conclusion is provided in section 5 .

\section{Theoretical Development}

The geometry of the problem is shown in Fig. 1. The radius of loop $i(i=1,2, \ldots, N)$ is $b_{i}$ and each loop is made of a perfectly conducting thin wire of radius $a_{i}$. Loop $i$ is excited by a delta voltage source at $\phi_{i}=0$. An $\exp (j \omega \mathrm{t})$ time convention is assumed throughout this paper and is suppressed for convenience.

\subsection{The Current Distribution and Input Impedance}

The current on each loop can be expanded in Fourier series as $[1-4]$

$$
I_{i}\left(\phi_{i}^{\prime}\right)=\sum_{n=-\infty}^{\infty} I_{n}^{i} e^{j n \phi_{i}^{\prime}}=I_{0}^{i}+2 \sum_{n=1}^{\infty} I_{n}^{i} \cos n \phi_{i}^{\prime} .
$$

Enforcing the boundary conditions on the surface of each loop, a system of linear equations are obtained as

$$
\mathbf{Z}_{n} \mathbf{I}_{n}=\mathbf{V}
$$

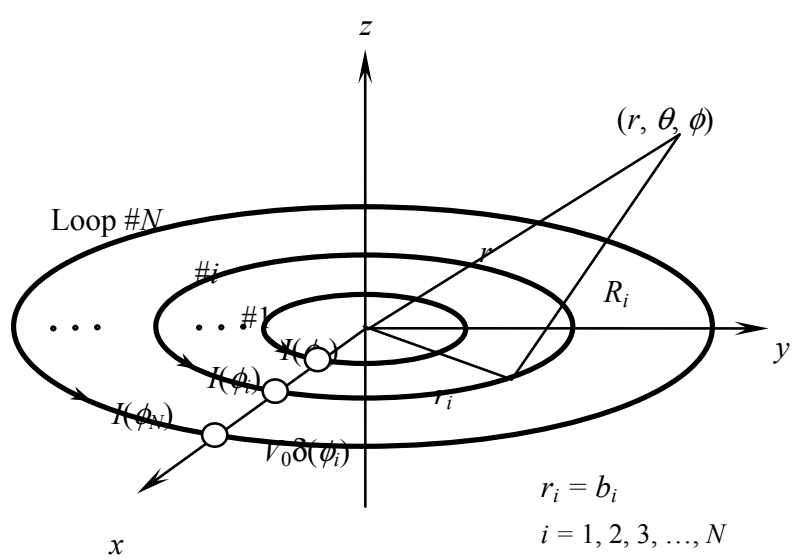

Figure 1: Geometry of the problem.

Where $\mathbf{V}$ is the excitation matrix with elements $V_{i} . \mathbf{I}_{n}$ is the current coefficient matrix to be determined and its elements are $I_{n}^{i} . \mathbf{Z}_{n}$ is the generalized impedance matrix. Since the loops are circular and coaxial, then elements of $\mathbf{Z}_{n}$ can be obtained using the same analysis as in [2] and [7] with the elements heights above $x-y$ plane are $d_{i}=0$, and $b_{i} \neq b_{j}$, where $(j=1,2, \ldots, N)$. Solving (2) for current coefficients then

$$
I_{n}^{i}=\sum_{j=1}^{N} y_{n}^{j i} V_{j}
$$

$y_{n}^{j i}$ are the elements of the matrix $\mathbf{Y}_{n}$, where

$$
\mathbf{Y}_{n}=\left(\mathbf{Z}_{n}\right)^{-1}
$$

The impedance at the input terminals of each loop can be obtained from (1) and (3) as

$$
Z_{i n}^{i}=\frac{V_{i}}{I_{i}\left(\phi_{i}^{\prime}=0\right)}=\frac{V_{i}}{\sum_{j=1}^{N} V_{j}\left(y_{0}^{j i}+2 \sum_{n=1}^{\infty} y_{n}^{j i}\right)} .
$$

\subsection{The Far-Fields and Directivity}

The Far-field expressions for CCLA can be obtained from that of an array of circular loops documented in [2] by substituting axial elements spacing $d_{i}=0$, then

$$
\begin{aligned}
& E_{\theta} \approx-\frac{\eta \cos \theta}{2} \frac{e^{-j \beta r}}{r} \sum_{i=1}^{N} \sum_{n=1}^{+\infty} j^{n} \beta b_{i} I_{n}^{i} \sin n \phi \\
& \times\left[J_{n-1}\left(u_{i}\right)+J_{n+1}\left(u_{i}\right)\right] \\
& E_{\phi} \approx \eta \frac{e^{-j \beta r}}{2 r} \sum_{i=1}^{N} \beta b_{i} I_{0}^{i} J_{1}\left(u_{i}\right) \\
&+\eta \frac{e^{-j \beta r}}{2 r} \sum_{i=1}^{N} \sum_{n=1}^{+\infty} j^{n} \beta b_{i} I_{n}^{i} \cos n \phi \\
& \\
& \times\left[J_{n+1}\left(u_{i}\right)-J_{n-1}\left(u_{i}\right)\right]
\end{aligned}
$$

Where $\beta$ is the wave number and $u_{i}=\beta b_{i} \sin \theta$. Assume $P_{i n}$ is the input power and no losses, then the directivity is

$$
D=2 \pi r^{2}\left(\left|E_{\theta}\right|^{2}+\left|E_{\phi}\right|^{2}\right) / \eta P_{i n} .
$$

\section{The Design Formulas}

Our goal is to present simple formulas that can be used to design CCLA antennas with a widest possible frequency band for $\mathrm{VSWR}<2$. The antenna is assumed to be driven through one of the loops while others are parasitic elements, since we are seeking a simple structure. If the driven element is loop $i$, then $V_{i}=V_{0}$ and $V_{j}=0$ for all $j \neq i$. The loops are of thin wires of thickness parameters $\Omega=\ln \left(2 \pi b_{i} / a_{i}\right)$. Under these conditions, (3) and (5) respectively, reduce to

$$
I_{n}^{i}=y_{n}^{j i} V_{i}
$$

and

$$
Z_{\text {in }}^{i}=1 /\left(y_{0}^{j i}+2 \sum_{n=1}^{\infty} y_{n}^{j i}\right)
$$

The VSWR based on a $50 \Omega$ line can be obtained from

$$
\operatorname{VSWR}=\frac{1+\left|\left(Z_{i n}^{i}-50\right) /\left(Z_{i n}^{i}+50\right)\right|}{1-\left|\left(Z_{i n}^{i}-50\right) /\left(Z_{i n}^{i}+50\right)\right|} .
$$


The antenna bandwidth for VSWR $<2$ can be computed using (4), (10) and (11).

\subsection{The CCLA}

The design steps for the broadband CCLA can be summarized as follows:

1) The design starts with a single resonant circular loop antenna (An\#1) of radius $b_{0}$. This antenna is driven by $50 \Omega$ line at the resonance frequency $\mathrm{f}_{\mathrm{o}}$. The thickness parameter for the loop is $\Omega=10$ and at the resonance frequency, $b_{0}$ is obtained as

$$
b_{0}=c_{0} / 2 \pi \mathrm{f}_{\mathrm{o}}
$$

Where $c_{0}$ is the speed of light in free space.

2) A parasitic loop with radius $b_{-1}<b_{0}$ is added to An\#1 to obtain a 2-elements CCLA (An\#2). To determine the value of $b_{-1}$ for maximum possible bandwidth, the bandwidth for $V S W R<2$ is computed for An\#2 using (9) - (11) as a function of $b_{-1}$ in the range $b_{-1}<b_{0}$ taking $\Omega=10$ for both elements. The variation of bandwidth against $b_{-1}$ is displayed in Fig. 2. The radius $b_{-1}$ for maximum bandwidth can be found from Fig. 2 as $b_{-1}=0.774 b_{0}$. In terms of $\mathrm{f}_{\mathrm{o}}$

$$
b_{-1}=0.774 c_{0} / 2 \pi \mathrm{f}_{\mathrm{o}} .
$$

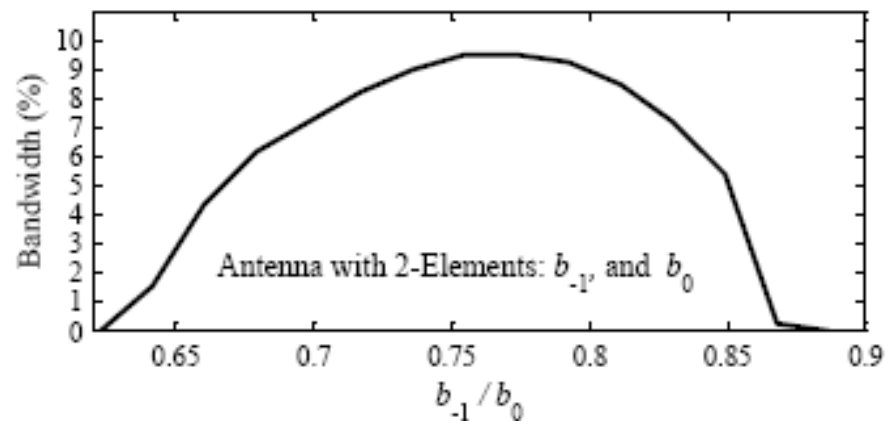

Figure 2: Variation of the bandwidth of a 2-element CCLA with $b_{-1}$.

3) A second parasitic loop with radius $b_{+1}>b_{0}$ is added to the An\#2 to obtain a 3-elements CCLA (An\#3). The bandwidth for $V S W R<2$ is re-computed using (9) - (11) for An\#3 as a function of $b_{+1}$ in the range $b_{+1}>b_{0}$ with $\Omega=10$ for all three elements and keeping $b_{0}$ and $b_{-1}$ at their previous values. The variation of bandwidth against $b_{+1}$ is displayed in Fig. 3. In this case, the value for $b_{+1}$ that gives best bandwidth for An\#3 is $b_{+1}=1.196 b_{0}$ as can be seen from Fig. 3, then

$$
b_{+1}=1.196 c_{0} / 2 \pi \mathrm{f}_{\mathrm{o}} .
$$

4) A 4-elements CCLA (An\#4) is obtained by adding a third parasitic loop with radius $b_{+2}>b_{+1}$ to An\#3. Similarly as above, the bandwidth for $\operatorname{VSWR}<2$ is re- computed using (9) - (11) for An\#4 as a function of $b_{+2}$ in the range $b_{+2}>b_{+1}$ keeping $b_{+1}, b_{0}$ and $b_{-1}$ at their previous values with $\Omega=10$ for all elements. In this case, the value for $b_{+2}$ that gives best bandwidth for An\#4 is $b_{+2}=2.83 \quad b_{0}$ as clear from Fig. 4 and can be written as

$$
b_{+2}=2.83 c_{0} / 2 \pi \mathrm{f}_{\mathrm{o}} .
$$

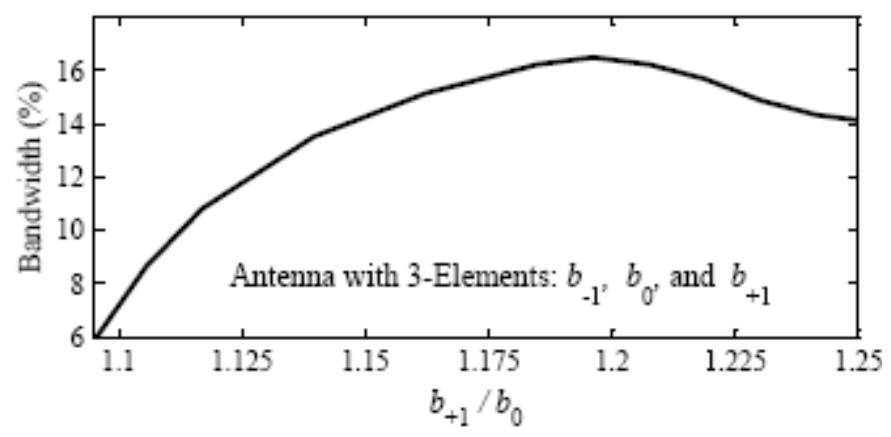

Figure 3: Variation of the bandwidth of a 3-element CCLA with $b_{+1}$.

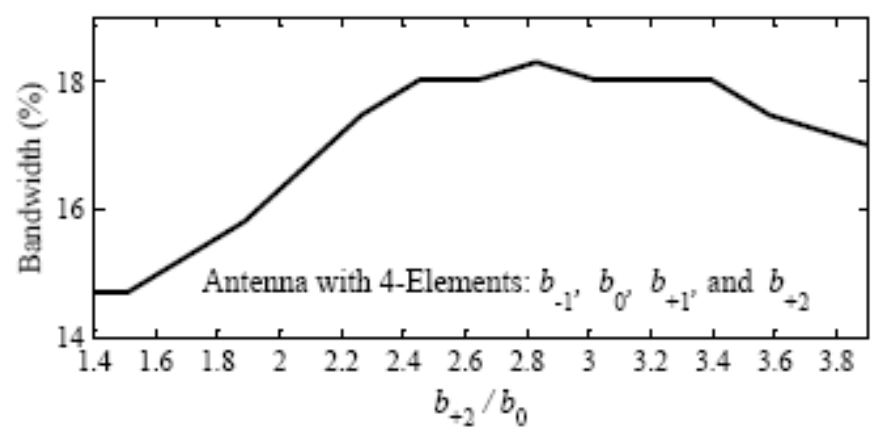

Figure 4: Variation of the bandwidth of a 4-element CCLA with $b_{+2}$.

5) More elements can be added with radii less than $b_{-1}$ or greater than $b_{+2}$ in a similar manner as in 2) to 4). However, the best bandwidth using this technique is determined mainly by the first four elements of radii $b_{-1}$, $b_{0}, b_{+1}, b_{+2}$. Additional elements may be used to improve the $V S W R$ around center frequency or increase the directivity. The outer element gives bandwidth $>$ $16 \%$ in the range $1.9<b_{+2} / b_{0}<4$ as shown in Fig. 4 .

Larger $b_{+2}$ increases the size and directivity of the antenna. So, $b_{+2}$ can be adjusted according to the size, directivity, and bandwidth requirements. Adding more elements may improve the directivity within the required bandwidth. However, this will increase the size of the antenna.

6) The center frequency of the antenna can be skewed to the right or left by scaling the dimensions of the antenna to the required frequency.

The procedure described in the above steps 1) to 6) indicates that when an element is added in each step, (9) - (11) are used to re-calculate the current distribution, input impedance, 
VSWR and bandwidth. This is necessary because these parameters depends on the number of elements and their dimensions. Various CCLAs are designed for best bandwidths using the design procedure listed in 1) to 6) steps. The optimal bandwidth for antennas with dimensions relative to the driven element are summarized in Table 1 . The bandwidth for $N=4,5$, and 6 elements are nearly the same as shown in Table 1, which indicates that there is no significant improvement in the VSWR when $N$ exceeds 4 elements. The computed VSWR for the first five antennas in Table 1 are plotted in Fig. 5 as a function of relative frequency $f / f_{o}$. The VSWR for a single loop is also superimposed to compare with multi-element antenna behavior. The VSWR $=2.0$ line is added to Fig. 5 to indicate desired operating bands.

Table 1: Sizes of different CCLA for best bandwidths.

\begin{tabular}{|c|c|c|c|c|c|c|c|}
\hline \multirow[t]{2}{*}{$N$} & \multirow[t]{2}{*}{$\begin{array}{l}\text { BW } \\
(\%)\end{array}$} & \multicolumn{6}{|c|}{$\begin{array}{l}\text { Radii of the elements relative to that of the } \\
\text { driven element }\left(b / b_{0}\right) \text { where } b_{0}=c_{0} / 2 \pi f_{0} \text { and } \\
\qquad \Omega=10\end{array}$} \\
\hline & & $b_{-2} / b_{0}$ & $b_{-1} / b_{0}$ & $b_{0} / b_{0}$ & $b_{+1} / b_{0}$ & $b_{+2} / b_{0}$ & $b_{+3} / b_{0}$ \\
\hline 2 & 9.61 & & 0.774 & 1.0 & & & \\
\hline 3 & 16.9 & & 0.774 & 1.0 & 1.196 & & \\
\hline 4 & 18.3 & & 0.774 & 1.0 & 1.196 & 2.83 & \\
\hline 5 & 18.3 & 0.236 & 0.774 & 1.0 & 1.196 & 2.83 & \\
\hline 6 & 18.4 & 0.236 & 0.774 & 1.0 & 1.196 & 2.83 & 3.019 \\
\hline
\end{tabular}

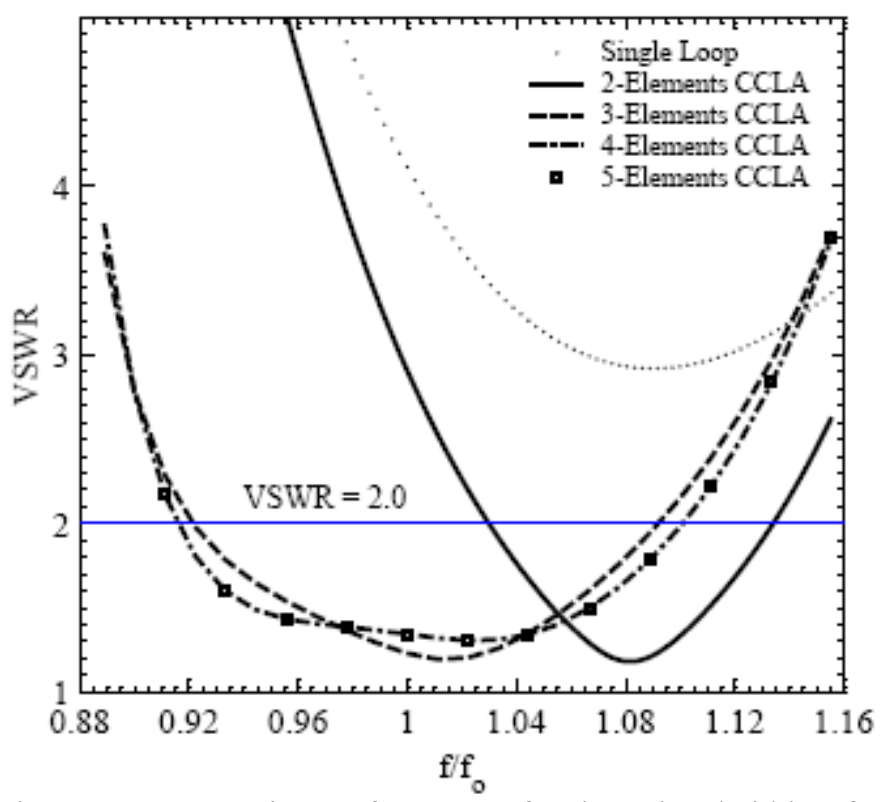

Figure 5: Comparison of VSWR for best bandwidth of different CCLAs with that of a single loop. The thickness parameter for each loop is $\Omega=10$.

\subsection{The CCLA Backed with a Ground Plane}

When the CCLA is backed with a perfectly conducting ground plane as shown in Fig. 6, the ground plane will affect the current distribution and the antenna characteristics. We assume that the ground plane is a square in shape of side $W$ and the spacing between it and the CCLA is $d$. A spacer of a honeycomb dielectric can be inserted between the ground plane and CCLA to support the structure of the antenna. The dielectric constant of this dielectric is $\varepsilon_{r} \approx 1$ at microwave frequencies. When the side of the ground plane $W$ satisfies both of the conditions: $W>5 b$ and $W>1.5 d$, where $b$ is the radius of the outer loop, it can be considered approximately infinite with respect to the loops [7]. In this case the image theory can be applied and the system of linear equations govern the current coefficients in (2) are

$$
\left[\begin{array}{ll}
\mathbf{Z}_{n}^{s s} & \mathbf{Z}_{n}^{s m} \\
\mathbf{Z}_{n}^{m s} & \mathbf{Z}_{n}^{m m}
\end{array}\right]\left[\begin{array}{l}
\mathbf{I}_{n}^{s} \\
\mathbf{I}_{n}^{m}
\end{array}\right]=\left[\begin{array}{l}
+\mathbf{V} \\
-\mathbf{V}
\end{array}\right]
$$

where $s$ for the loops and $m$ for images of the loops. Since $\mathbf{Z}_{n}^{s s}=\mathbf{Z}_{n}^{m m}, \mathbf{Z}_{n}^{m s}=\mathbf{Z}_{n}^{m s}$ and $\mathbf{I}_{n}^{m}=-\mathbf{I}_{n}^{s}$, then (16) reduces to

$$
\left(\mathbf{Z}_{n}^{s s}-\mathbf{Z}_{n}^{s m}\right) \mathbf{I}_{n}^{s}=\mathbf{V}
$$

The elements of $\mathbf{Z}_{n}^{s s}$ and $\mathbf{Z}_{n}^{s m}$ can be obtained using the same procedure followed for $\mathbf{Z}_{n}$ in (2) with $d_{i}=d$ for the loops and $d_{i}=-d$ for the images. Once $\mathbf{I}_{n}^{s}$ is determined from (17), then characteristics of the CCLA backed with a ground plane can be obtained. The far-fields components in this case can be obtained by superimposing fields from the loops and their images.
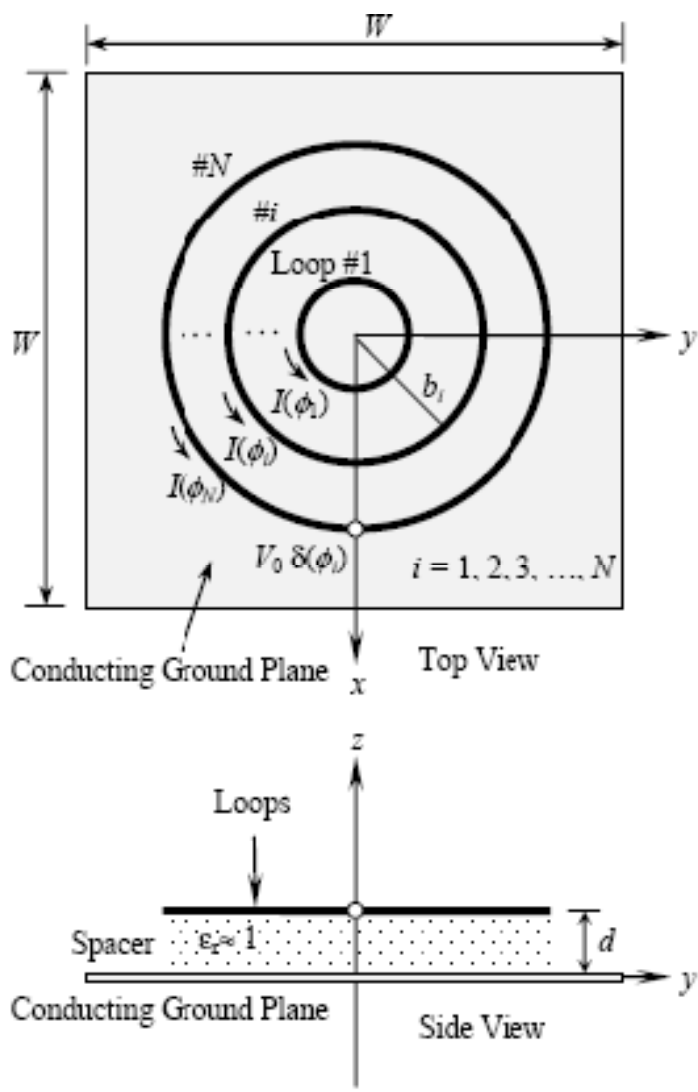

Figure 6: Geometry of the CCLA backed with a conducting ground plane.

The far-fields components from the loops and their images 
can be obtained in a similar manner as in (6)and (7), then for CCLA above the ground plane the far-fields components are

$$
\begin{gathered}
H_{\theta} \approx-j \sin (\beta d \cos \theta) \frac{e^{-j \beta r}}{r} \sum_{i=1}^{N} \beta b_{i} I_{0}^{i} J_{1}\left(u_{i}\right) \\
-j \sin (\beta d \cos \theta) \frac{e^{-j \beta r}}{r} \sum_{i=1}^{N} \sum_{n=1}^{+\infty} j^{n} \beta b I_{n}^{i} \\
\times\left[J_{n+1}\left(u_{i}\right)-J_{n-1}\left(u_{i}\right)\right] \cos n \phi \\
H_{\phi} \approx-j \cos \theta \sin (\beta d \cos \theta) \frac{e^{-j \beta r}}{r} \\
\times \sum_{i=1}^{N} \sum_{n=1}^{+\infty} j^{n} \beta b_{i} I_{n}^{i} \\
\times\left[J_{n-1}\left(u_{i}\right)+J_{n+1}\left(u_{i}\right)\right] \sin n \phi
\end{gathered}
$$

where $u_{i}=\beta b_{i} \sin \theta$. The directivity and VSWR of the CCLA backed with a ground plane can be obtained from (8) and (11) using (16) - (19) respectively.

The analysis shows that a 3-elements CCLA backed with a ground plane as shown in Fig. 6 can give a broadband characteristics with high directivity. In this case, the radii are $b_{-2}, b_{-1}$ and $b_{0}$ and $d$ is the antenna height above the ground plane. Taking $b_{0}$ as a driven element, the radii for the best bandwidth are:

$$
\left\{\begin{array}{l}
b_{0}=c_{0} / 2 \pi \mathrm{f}_{\mathrm{o}}, b_{-1}=0.832 b_{0}, b_{-2}=0.678 b_{0} \\
d=1.038 b_{0}
\end{array}\right.
$$

The VSWR for the CCLA of dimensions given in (20) is shown in Fig. 7 as a function of normalized frequency $f / f_{0}$ for different thickness parameters. The thickness parameters of the loops have a significant effect on the VSWR and bandwidth of the CCLA as can be seen from Fig. 7. Small changes in the thickness parameter may results in a significant bandwidth variation.

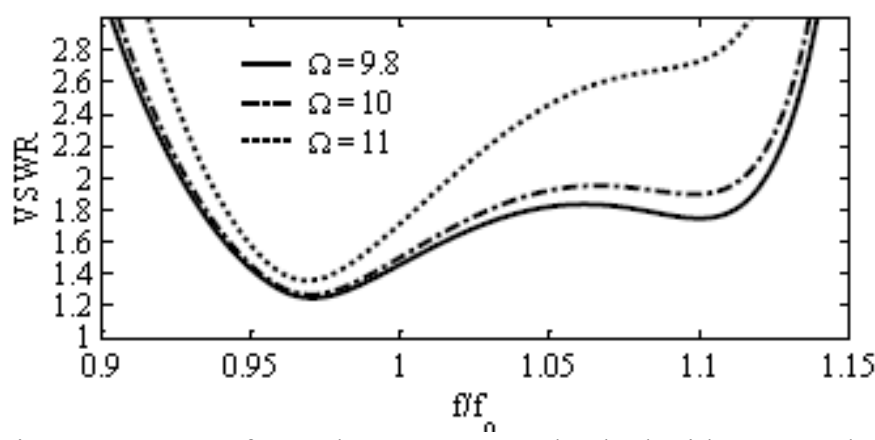

Figure 7: VSWR for 3-element CCLAs backed with a ground plane. The height above the plane is $d=1.0384 b_{0}$.

\section{Numerical Results}

This section presents results with discussion for CCLAs in the UHF and microwave frequency bands using the formulation obtained in the previous sections. Both the bandwidth and the radiation characteristics for different
CCLAs are discussed.

\subsection{CCLA for Broadband UHF Applications}

The CCLA can be designed for far-field RFID applications as a reader antenna. Referring to Fig. 5, the 4-elements CCLA gives a fractional bandwidth of $18.3 \%$ within the range $0.916<\mathrm{f} / \mathrm{f}_{\mathrm{o}}<1.101$, while 3 -element CCLA gives a fractional bandwidth of $16.9 \%$. These antennas can be designed to cover all the frequency bands for UHF RFID applications, which are extended from $840 \mathrm{MHz}$ to $960 \mathrm{MHz}$. By choosing $\mathrm{f}_{\mathrm{o}}=900 \mathrm{MHz}$, the frequency band satisfies VSWR $<2$ for 4-elements antenna is $825 \mathrm{MHz}<\mathrm{f}<991$

\begin{tabular}{|c|c|c|c|c|c|c|c|c|}
\hline \multirow[t]{2}{*}{$N$} & \multirow[t]{2}{*}{$\begin{array}{l}D_{\min } \\
(d B)\end{array}$} & \multirow[t]{2}{*}{$\begin{array}{l}\text { BW } \\
\text { (MHz) }\end{array}$} & \multicolumn{6}{|c|}{$\begin{array}{l}\text { Radii of the elements in } \\
\text { cm(Thickness parameter for all } \\
\text { elements is } \Omega=10 \text { ) }\end{array}$} \\
\hline & & & $b_{-2}$ & $b_{-1}$ & $b_{0}$ & $b_{+1}$ & $b_{+2}$ & $b_{+3}$ \\
\hline & 3.5 & 93.6 & & 4.1 & 5.3 & & & \\
\hline 3 & 3.9 & 153.3 & & 4.1 & 5.3 & 6.34 & & \\
\hline 4 & 5.2 & 166.2 & & 4.1 & 5.3 & 6.34 & 15 & \\
\hline 5 & 5.2 & 165.8 & 1.25 & 4.1 & 5.3 & 6.34 & 15 & \\
\hline 6 & 5.5 & 167.0 & 1.25 & 4.1 & 5.3 & 6.34 & 15 & 16 \\
\hline
\end{tabular}
$\mathrm{MHz}$. The radius of the driven element at $900 \mathrm{MHz}$ from (12) is $b_{0}=5.3 \mathrm{~cm}$ and the other elements can be computed using (13) - (15). The radii for the different CCLAs for the best bandwidth (BW) are summarized in Table 2 with the minimum directivity $\mathrm{D}_{\min }$ in each band.

Table 2: Different CCLAs in the UHF RFID bands.

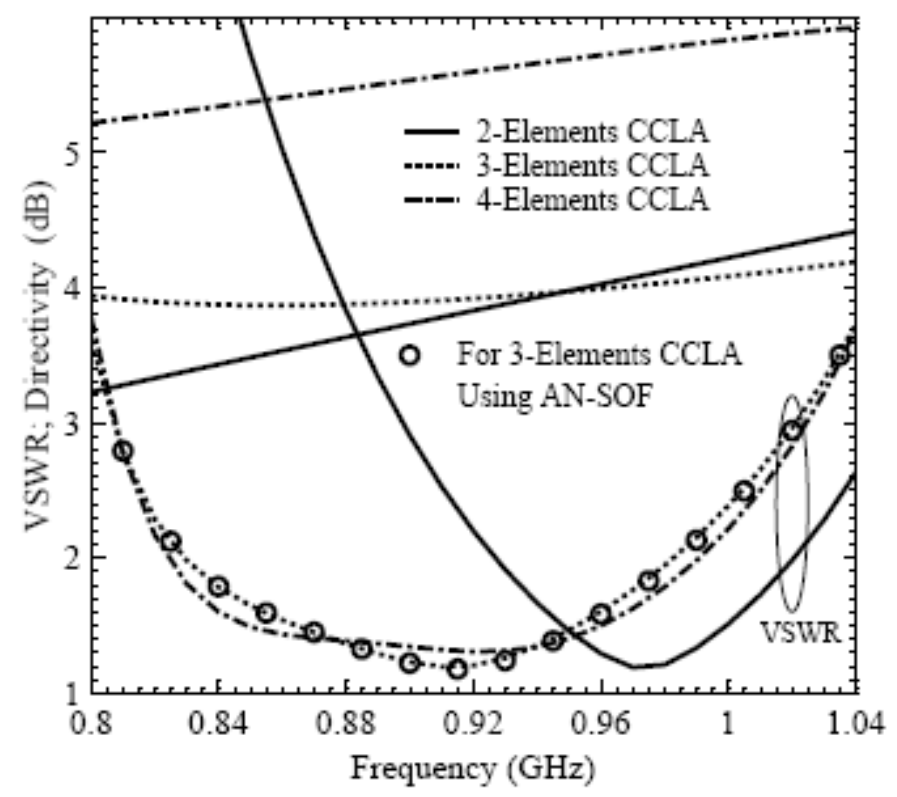

Figure 8: VSWR and directivity of broadband CCLAs.

The VSWR and directivity for 2-, 3- and 4-elements CCLA are computed and displayed in Fig. 8 as a function of frequency. The results for the 3-elements case using AN-SOF software is also obtained and displayed in Fig. 8 to check the analytical results. Both the analytical results and that from AN-SOF are in excellent agreement. Furthermore, Fig. 8 shows that the bandwidth of the 3-and 4-elements antennas covers the whole UHF RFID frequency band (840-960) $\mathrm{MHz}$ 
and the minimum directivities in this band for the 3-elements and 4-elements antennas are about $3.9 \mathrm{~dB}$ and $5.2 \mathrm{~dB}$ respectively. The directivity for 4-elements CCLA can be increased over $6 \mathrm{~dB}$ by adjusting the size of the outer element. In fact, the radius of the outer element $b_{+2}$ has a wide range of choices based on the required directivity, antenna size, and bandwidth as shown in Fig. 9. Moreover, Fig. 9 shows that, the directivity is nearly stable in the band (840-960) $\mathrm{MHz}$ for values of $b_{+2}$ extending from $7 \mathrm{~cm}$ to 22 $\mathrm{cm}$. For the range $26 \mathrm{~cm}<b_{+2}<30 \mathrm{~cm}$, the directivity at 960 $\mathrm{MHz}$ decreases to less than $3 \mathrm{~dB}$ while it is above $3.8 \mathrm{~dB}$ at $840 \mathrm{MHz}$.

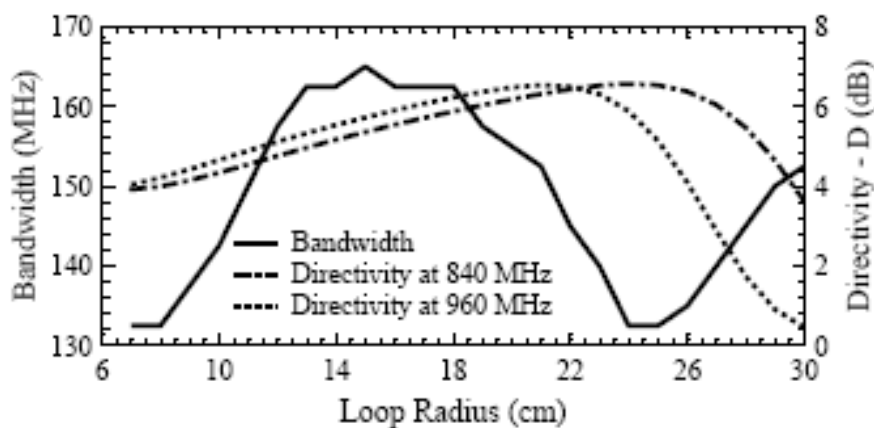

Figure 9: The effect of $b_{+2}$ on the directivity and bandwidth of 4-elements CCLA.
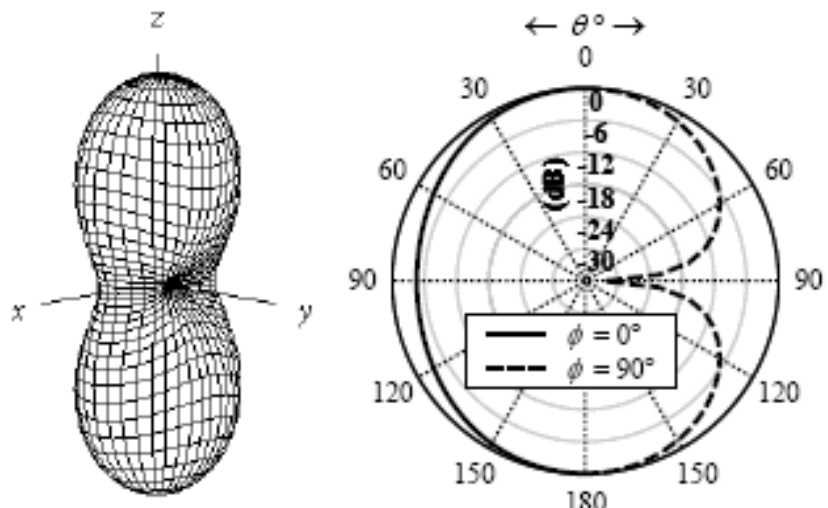

Figure 10: Radiation patterns of the 3-elements CCLA at 900 MHz.
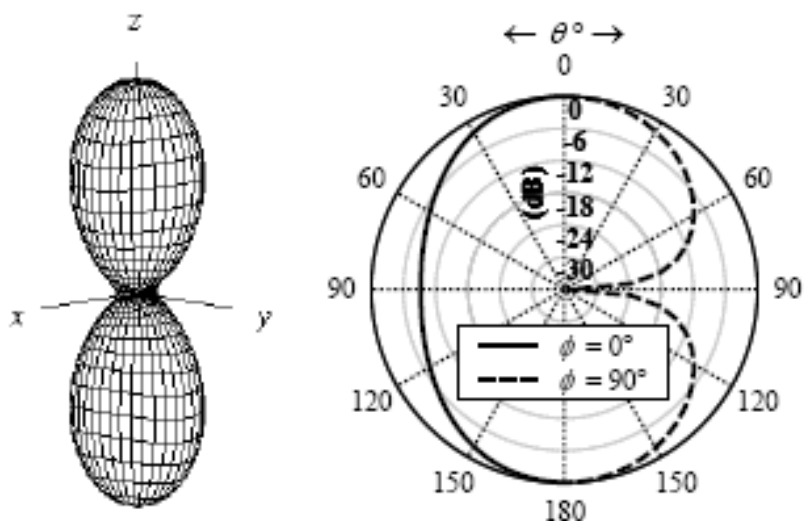

Figure 11: Radiation patterns of the 4-elements CCLA at 900 $\mathrm{MHz}$.

The radiation patterns at $900 \mathrm{MHz}$ for 3-elements and 4elements CCLA of dimension given in Table 2 are displayed in Figs. 10 and 11 respectively. The general shape of the pattern for both antennas is similar to that of the resonant single loop antenna. However, the beam-width of the 3elements antenna is wider than that of 4-elements. The halfpower beam-width (HPBW) and the maximum directivity (D) in the forward direction for both antennas and their dimensions are shown in Table 3.

Table 3: Radiation patterns of 3- and 4-elements CCLA at $900 \mathrm{MHz}$

\begin{tabular}{cccccccc}
\multirow{N}{N}{} & \multirow{2}{*}{$\mathbf{D}(\mathrm{dB})$} & \multicolumn{2}{c}{$\begin{array}{c}\text { HPBW in } \\
\text { planes }\end{array}$} & \multicolumn{4}{c}{$\begin{array}{c}\text { Radii of the elements } \\
(\mathbf{c m})\end{array}$} \\
\cline { 3 - 8 } & & $\boldsymbol{\phi}_{\mathbf{0}}^{\circ}$ & $\boldsymbol{\phi}=\mathbf{9 0}^{\circ}$ & $\boldsymbol{b}_{\boldsymbol{- 1}}$ & $\boldsymbol{b}_{\mathbf{0}}$ & $\boldsymbol{b}_{\mathbf{+ 1}}$ & $\boldsymbol{b}_{+\mathbf{2}}$ \\
\hline 3 & 3.92 & $111^{\circ}$ & $81^{\circ}$ & 4.1 & 5.3 & 6.34 & \\
\hline 4 & 5.35 & $73^{\circ}$ & $75^{\circ}$ & 4.1 & 5.3 & 6.34 & 15 \\
\hline
\end{tabular}

\subsection{CCLA Backed with Ground Plane at Microwave Frequencies}

The parameters and dimensions for designing a 3-elements CCLA backed with a finite conducting ground plane are determined in section 3.2. Referring to Fig. 7, this antenna gives a fractional bandwidth of $19.6 \%$ within the range 0.926 $<\mathrm{f} / \mathrm{f}_{\mathrm{o}}<1.122$. By choosing $\mathrm{f}_{\mathrm{o}}=2.45 \mathrm{GHz}$, the frequency range in $\mathrm{MHz}$ for VSWR $<2$ can be obtained as $2.27 \mathrm{GHz}<$ $\mathrm{f}<2.75 \mathrm{GHz}$. From (20), the radius of the driven element at $2.45 \mathrm{GHz}$ is $b_{0}=19.5 \mathrm{~mm}$ and the other elements are: $b_{-1}$ $=16.1 \mathrm{~mm}, b_{-2}=13.2 \mathrm{~mm}$ and $d=20.2 \mathrm{~mm}$. The directivity, VSWR and the radiation pattern of the antenna are displayed in Figs. 12 and 13 respectively. The results for the VSWR using AN-SOF software are also obtained and displayed in Fig. 12 to check the analytical results. The analytical results and that from AN-SOF are in excellent agreement.

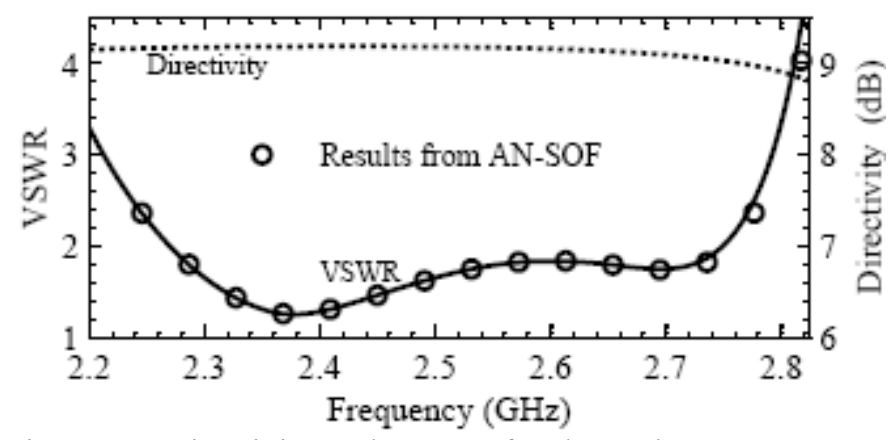

Figure 12: Directivity and VSWR for the 3-elements CCLA backed with ground plane with $b_{-2}=13.2 \mathrm{~mm}, b_{-1}=16.1 \mathrm{~mm}$, $b_{0}=19.5 \mathrm{~mm}, d=20.2 \mathrm{~mm}$, and $\Omega=9.8$.

The CCLA backed with ground plane gives a stable directivity of about $9.15 \mathrm{~dB}$ over the entire bandwidth of interest. Using the conditions discussed in section 3.2, the ground plane of side $W=26.5 \mathrm{~cm}$ can be considered as an infinite ground plane to the loops. It is obvious that the radiation pattern of the CCLA backed with a ground plane to be unidirectional as shown in Fig. 13. The HPBW in the plane of $\phi=0^{\circ}$ is $77.9^{\circ}$ and in the plane $\phi=90^{\circ}$ is $67.3^{\circ}$. The bandwidth for VSWR $<2$ and dimensions of the CCLA backed with a ground plane at $2.45 \mathrm{GHz}$ and $5.8 \mathrm{GHz}$ are shown in Table 4. 

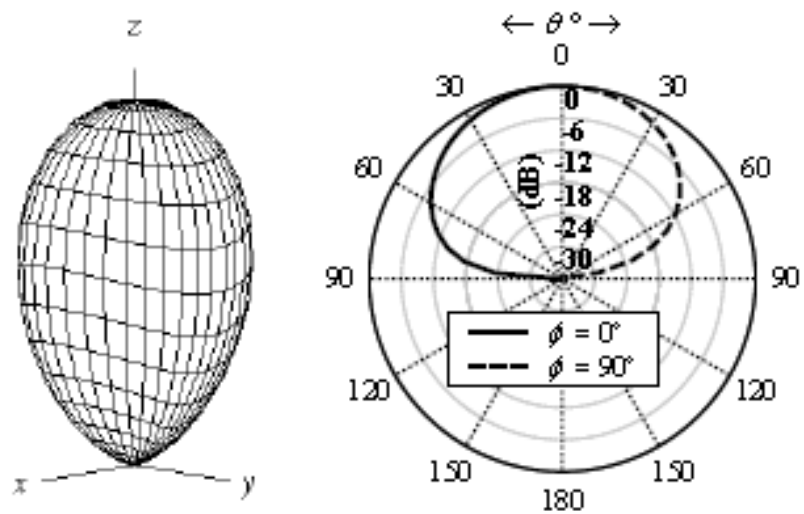

Figure 13: Radiation patterns for the 3-element CCLA backed with ground plane with $\beta b_{0}=1$. and $d=1.0384 b_{0}$.

Table 4: 3-Elements CCLAs backed with ground planes

\begin{tabular}{|c|c|c|c|c|c|c|}
\hline \multirow{2}{*}{$\begin{array}{c}\mathbf{f}_{\mathbf{o}} \\
(\mathbf{G H z})\end{array}$} & \multirow{2}{*}{$\begin{array}{c}\text { BW } \\
\text { RANGE } \\
\text { (GHz) }\end{array}$} & \multicolumn{5}{|c|}{ Dimensions (mm) } \\
\hline & & $b_{-2}$ & $b_{-1}$ & $b_{0}$ & $d$ & $W$ \\
\hline 2.45 & $2.27-2.75$ & 13.2 & 16.1 & 19.5 & 20.2 & 97.4 \\
\hline 5.80 & $5.37-6.51$ & 5.6 & 6.9 & 8.2 & 8.6 & 41.1 \\
\hline
\end{tabular}

\section{Conclusions}

Theoretical formulations that can be used to design broadband concentric circular-loop antennas are presented in this paper. The formulations are employed to design various CCLAs with and without ground planes at UHF and microwave frequency bands. For the CCLAs without ground plane, a fractional bandwidth of $18.3 \%$ can be obtained for VSWR $<2$ with directivity higher than $5.2 \mathrm{~dB}$ in the entire bandwidth. The CCLA backed with a finite conducting ground plane can be designed to operate in a wider band of fractional bandwidth of $19.6 \%$ and directivity of $9.15 \mathrm{~dB}$ within the entire band of interest. The AN-SOF antenna simulation software is used to validate analytical results where an excellent agreement between analytical and simulated results is observed. The main features of the CCLAs, that they are simple, low cost, and can be designed to cover the universal frequency band for UHF RFID applications.

\section{References}

[1] G. S. Smith, "loop antennas," in Antenna Engineering Handbook, R. C. Johnson and H. Jasik, Eds. New York: McGraw-Hill, ch. 5, 2007.

[2] S. Ito, N. Inagaki, and T. Sekiguchi, Investigation of the array of circular-loop antennas, IEEE Trans. Antennas Propagat. 4: 469-476, 1971.

[3] R. W. P. King, The loop antenna for transmission and reception, In Antenna Theory, Part I, Collin, R. E. and Zucker, F. J. New York: McGraw-Hill, 1969.

[4] R. W. P. King and G. S. Smith, Antenna in Matter: Fundamentals, Theory and Applications, Cambridge, MA: MIT press, pp. 527-569, 1981.

[5] C. A. Balanis, Antenna Theory, Analysis, and Design, New York: Harper and Row, ch. 5, 1982.

[6] A. Shoamanesh, and L. Shafei, Characteristics of circular loop antenna above a lossless ground plane,
IEEE Trans. Antennas Propagat. AP-29: 528-529, 1981.

[7] H. A. N. Hejase, S. D. Gedney, and K. W. White, Effect of a finite ground plane on radiated emission of a circular loop antenna, IEEE Trans. Electromagn. Compat. EMC-36: 364-371, 1994.

[8] B. S. Hoon, L. Byungje, and J. H. Frances, Multi-band internal loop antenna with inserted concentric rings for mobile terminals, in Proc., IEEE Antennas and Propagat. Int. Symposium, pp. 4725-4728, 2007.

[9] R. L. Li, G. DeJean, J. Laskar, and M. M. Tentzeris Investigation of circularly polarized loop antennas with parasitic element for bandwidth enhancement, IEEE Trans. Antennas Propagat. AP-53: 3930-3939, 2005.

[10] H. Nakano, M. Fukasawa, and J. Yamauchi, Discrete multiloop, modified multiloop, and plate-loop antennasmultifrequency and wide-band VSWR characteristics, IEEE Trans. Antennas Propagat. AP-50: 371-378, 2002.

[11] S. M. A. Hamed and M. A. H. Abbas, Radiation from a circular loop antenna placed coaxially relative to a conducting body of revolution, IEEE Trans. Antennas Propagat. AP-60: 4937- 4940, 2012.

[12] S. M. A. Hamed and S. O. Bashir, Characteristics of a circular loop in the presence of a coaxial conducting BOR attached to a planar Reflector, IEEE Antennas and Wireless Propagat. Letters 12: 793 - 796, 2013.

[13] A. C. Schell and E. L. Bouche, A concentric loop array, in Proc. WESCON, pp. 212-218, 1958.

[14] Z. N. Chen, X. Qing and H. L. Chung, A universal UHF RFID reader antenna, IEEE Trans. Microwave Theory and Techniques 57: 1275- 1282, 2009.

[15] X. Qing, C. K. Goh and Z. N. Chen, A broadband UHF near-field RFID antenna, IEEE Trans. Antennas Propagat. AP-58: 3829- 3838, 2010.

[16] X. Li and J. Liao, Eye-shaped segmented reader antenna for near-field UHF RFID applications, Progress In Electromagnetics Research 114: 481- 493, 2011.

[17] S. Fan, S. Zheng, Y. Cai, Y. Yin, Y. Hu, and J. Yang, Design of a novel wideband loop antenna with parasitic resonators, Progress In Electromagnetics Research Letters 37: 47- 54, 2013.

[18] Y. Li, Z. Xie and X. Cen, Compact loop antenna for near-field and far-field UHF RFID applications, Progress In Electromagnetics Research C 37: 171- 182, 2013.

[19] P. Wang, G. Wen, J. Li, Y. Huang, L. Yang and Q. Zhang, Wideband circularly polarized UHF RFID reader antenna with high gain and wide axial ratio beamwidths, Progress In Electromagnetics Research 129: 365- 385, 2012.

[20] X. Lai, J. Ouyang and P. Yang, A circularly polarized compact antenna for UHF band RFID reader, Progress In Electromagnetics Research Letters 42: 119- 127, 2013.

[21] J. Uddin, M. B. I. Reaz, M. A. Hasan, N. A. Nordin, M. I. Ibrahimy and M. A. M. Ali, UHF RFID antenna architectures and applications, Scientific Research and Essays 5(10): 1033- 1051, 2010. 